\title{
Contemporary Interpreting and Innovative Design of Rune Characters under Cultural Creative Thinking*
}

\author{
Zhixiong Huang \\ Fuzhou University \\ Xiamen, China
}

\begin{abstract}
Rune, a kind of ancient culture, is the typical representative of China's Taoism calligraphy, boasting unique aesthetics features and mysterious cultural connotation. This paper conducts analyses on rune's character modeling features, form language, structural framework, symbolic features and aesthetic value, offers some advice that re-design of rune character should consider both interestingness and beauty, highlight practicability in combination with principles to develop creative products, and conduct research on the feasibility to search for appropriate carriers for developing cultural products in combination with modern design on the basis of retaining rune characters' modeling features, and thus explores contemporary interpreting and innovative design mode of folk custom culture of the same kind and tries to find a broader consumption market.
\end{abstract}

Keywords-cultural creative thinking; rune; character modeling; innovative design

\section{INTRODUCTION}

Taoistic rune can trace back to the ancient wizard at the earliest. There is historical evidence proving that rune is very popular in Eastern Han Dynasty when almost each household owns rune to drive out evil spirits and introduce good luck. "Rune" comes down in one continuous line with linguistic worship. When Taoist priests are drawing runes or using runes, there are accompanying corresponding incantations. The important status of incantation in Taoism is related to the original linguistic worship. In the primitive society, language is used to issue orders, achieving unified actions in hunting. Therefore, people think that language is a kind of magical skill given by god, through which communication with the god can be achieved to introduce good luck and drive out disasters. Language worship gradually is centrally reflected in incantation. Taoistic incantation usually starts with an edict and ends with "as urgent as a legal order", which is inherited from Master Zhang of Han Dynasty to ask the god to take actions according to the rune, hoping to receiving the god's effective response immediately upon arrival of the rune. Later on, oral incantation becomes written with the appearance of characters and evolution of written carrier, thus "rune" appears. The paper mainly conducts research on lettering and praying's cultural connotation of rune and discusses the possibility to develop the mysterious rune culture in combination with contemporary culture creative thinking.

\section{WRITING FRAMEWORK OF RUNE}

Frame of rune falls into five parts "Fig. 1": rune heading, God and Buddha managing the affairs, rune belly, rune gallbladder and rune feet. (1) Rune: Just as its name implies, rune heading is located at the top with three ticks representing three supernatural beings "Fig. 2". To achieve effective responses, there should be corresponding incantations accompanying writing of the three ticks. Some rune headings omit the three ticks and take an "edict" as the replacement "Fig. 3". (2) God and Buddha managing the affairs: generally written above the edict and beneath the three ticks. They are in big character, so do rune heading and rune gallbladder. (3) Rune belly: above or beneath the edict. The content of rune gallbladder describes the theme of the rune and gods to order about. It is generally in small character sometimes with corresponding diagrams to intensify narrative effect. (4) Rune gallbladder: the key to determine whether a rune can fully play its role. It is the soul of the whole rune, equaling to inviting the god to enter into the rune. (5)Rune feet: at the bottom of the rune and of extreme importance. Some runes may only have three parts: rune heading, rune belly and rune feet, like dystocia rune and Dhammuttariya rune in urgency. Due to its framework, rune is mainly in strip form with well-proportioned characters of different sizes.

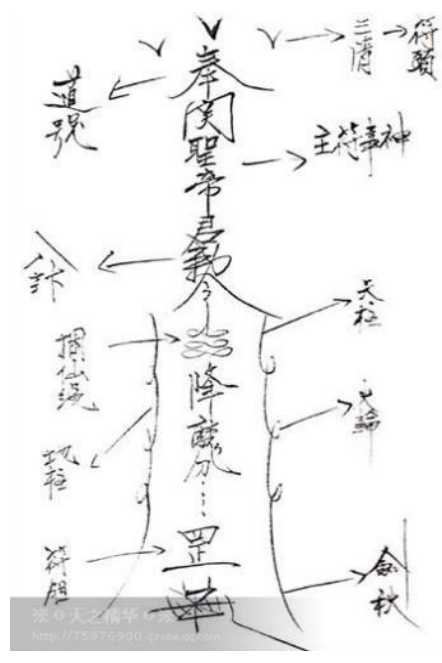

Fig. 1. Structure Diagram of Rune.

*Fund Project: 2016 Fujian Provincial Social Science Plannin Project(FJ2016B215). 


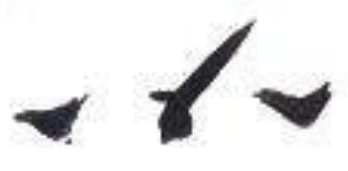

Fig. 2. Three Sticks.

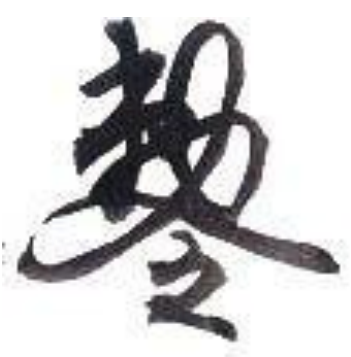

Fig. 3. Edict.

\section{AESTHETIC CHARACTERISTICS OF RUNE}

\section{A. Common Origins of Calligraphy and Painting in Rune}

Rune is the typical representative of China's Taoistic calligraphy, whose typeface originates from traditional seal character. Through exaggerated transformation, regulation of strokes and adding of star, drifting cloud and ghosts and gods symbols, the new runes come into being with excellent pictures and accompanying essays in flexion as a whole. Besides the function of carrying and conveying information, rune boasts unique modeling art with rich implied meaning, characters containing the picture and picture containing characters. This kind of manifestation mode and common origins of calligraphy and painting bring out the best in each other. Then, the rune is written on yellow laminate, silk or wooden plate with zinnober or ink. Due to the special character frame and formal language, it has unique aesthetic value. Taoistic rune originates from calligraphy and exerts a good effect on calligraphy and its character formation mode and framework have great influence on calligraphy in Tang Dynasty. The wild scribble of Zhang Xu, a famous calligrapher, directly benefits from the wild and cathartic Taoistic rune. In his Highlights of Chinese Calligraphy History, Tao Zongyi in Ming Dynasty praises, "His calligraphy is characterized by hollow strokes with momentum of writing, which can't be learnt". ${ }^{1}$ The technique of character transformation still is of great reference importance to today's character design. Besides, the Qi theory in taoistic rune changes the thinking mode of calligraphy and plays an important facilitating role in creation of rune characters.

\section{B. Rune Stroke Characteristics}

The biggest difference between characters on the rune and printing character lies in that rune character is manuscript and random while the latter is orderly and upright with evident laws. Form is bound to serve the function. For the convenience

${ }^{1}$ Xie Changyong. Discussion on Taoism's Influence on the Art of Painting and Calligraphy of Tang Dynasty [J]. Art Panorama, Issue 8, 2008, Page 31. of massive replication, there are high requirements for identification and normalization of printing character with relative few changes. As Taoistic arts of necromancy, rune is mysterious and can't be passed on to others. Its writing is done in line with execration ceremony. Determined by rune makers' mood in writing and way to wield the pen, strokes are more random and changeful. However, it doesn't mean that no trace can be found in stroke characteristics and laws. The paper conducts research and in-depth analyses on character strokes' features, structure, combination and decoration and conducts integration of features and transformation laws.

\section{1) The major role of distortion in writing}

As to the process of rune writing, it is particular about finishing writing at one stretch in pursuit of Taoistic "Qi" and the cloud-like artistic conception of Yun Zhuan Tian Shu, therefore distortion plays the major role in writing, which is tightly related to and comes down in one continuous line with the taoistic theory that man is an integral part of nature. The rune heading and feet are written in distortion to endow the rune with a sense of form and mystery as shown in "Fig. 4" and "Fig. 5".

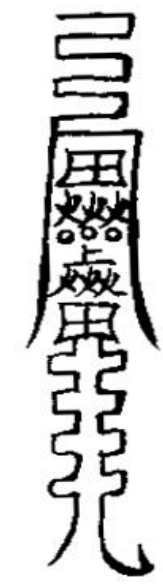

Fig. 4. Good and Bad Omen Rune.

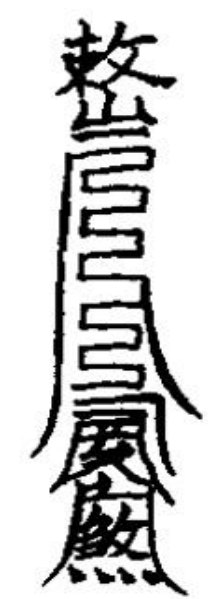

Fig. 5. Direction God Rune Ru. 


\section{2) The subsidiary role of joined-up writing}

Taoist priests incant and draw the rune quickly at the same time, therefore inevitably there are many joined-up strokes, which sometimes appear in the same character and sometimes between two characters. For example, the last stroke of "Chi" and the first stroke of "Ling" are joined up in Chinese characters "Chi Ling"(edict). In addition, influenced by cursive script's way of writing, some joined-up strokes appear. The healing rune in "Fig. 6" is drawn under water, therefore it is completed at one stretch, manifesting the joined-up stroke vividly.

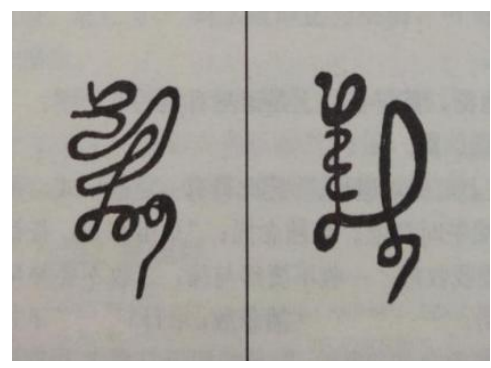

Fig. 6. Emesis Rune and Diarrhea Rune.

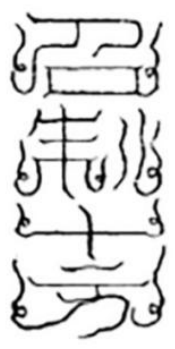

Fig. 7. Decorative Strokes.

\section{3) Increase and Decrease of strokes}

Every rune has its specific meaning. Sometimes increase and decrease of strokes is adopted to emphasize the effect of narrative. For example, the Chinese character "Gui" (ghost) omits the first stroke as shown in "Fig. 7" on the premise of ensuring identifiability, meaning the ghost is cracked down.

\section{4 ) Decorative strokes}

Decorative strokes are the formal language enriching the whole rune and an important element adding a sense of mystery. The common ways are as follows: lengthen or shorten strokes, convert straight strokes into curved strokes and vice versa, add curved strokes lingering to and fro in some strokes or add small dots. As shown in Picture 7, every character of "Zhao Zhi Shi Fang"has a curved stroke knotted upwards besides it, which acts as a decorative stroke without specific meaning. Some decorative strokes can complement the meaning of rune while some purely appear out of the need of formal sense without any meaning.

\section{5) Borrowing and comparison in combination}

Rune explains profound theories in simple language in the square cun paper of vertical rectangle. It is necessary to consider the borrowing relation and size comparison of characters to arrange the numerous and complicated content inside the rectangle. The way of combination acts as an excellent reference for contemporary character design.

\section{SYMBOLIC CHARACTERISTICS OF RUNE}

\section{A. Repeated Constitution of Character Pattern}

The repeated constitution of character in rune has the function of repeated narrative and emphasizing. In rune, it is common to see repeated usage of "Ji" (auspicious) and "Ri" (sun). "Ri" symbolizes the sun with masculinity, healing, driving out ghosts and evil spirits, and introducing good luck. In "Fig. 8", repeated appearance of "Li" (power) is for the purpose of adding efficacy to "Ri", changing their balance of power and pressing "ghosts" underneath. Thus, the whole family is auspicious with everything going well. In "Fig. 9", the repeated usage of "Huo" (fire) is also for the purpose of repeated narrative and emphasis, using "Huo" to subdue monsters and drive out ghosts. Character of repeated constitution balances the rune and increases the efficacy of form sense, its meaning making the rune interesting.

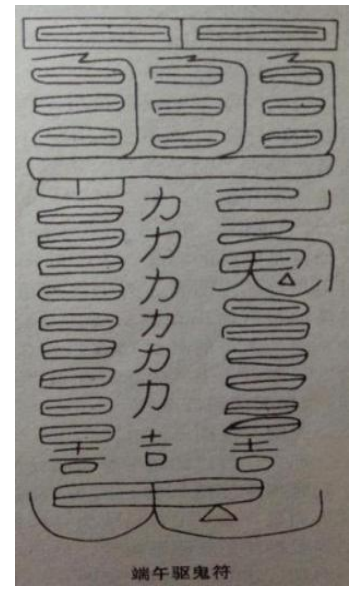

Fig. 8. Dragon Boat Festival Exorcism rune.

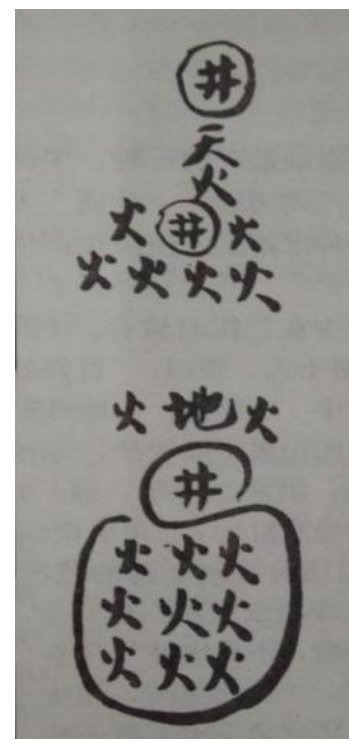

Fig. 9. Evil Spirits Control Rune. 


\section{B. Constitution of Square and Circle in Star Runes}

The Plough rune can subdue monsters and drive out ghosts, therefore it is usually used as amulet. Abstract the Big Dipper as a circle or a square, arrange and combine according to the meaning and partially link by thin lines to constitute the constellation. In "Fig. 10", the seven circles in the third part of the rune represent the Big Dipper with five stars in the middle and one at each side(totally two sides), totally seven stars. Some runes are directly named as "Seven-star rune". ${ }^{2}$ In "Fig. 11 ", scattered small blockages in the animal year rune represents stars, making it a star rune.

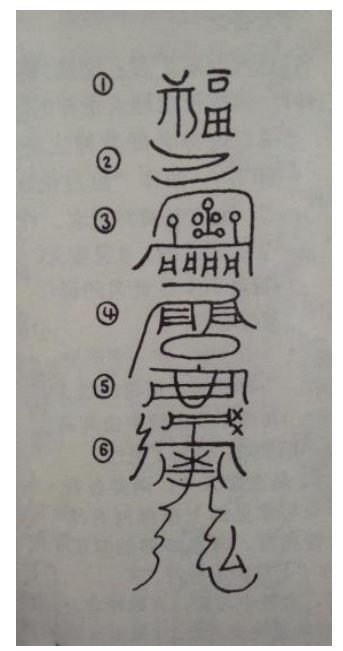

Fig. 10. Turpan Amulet.

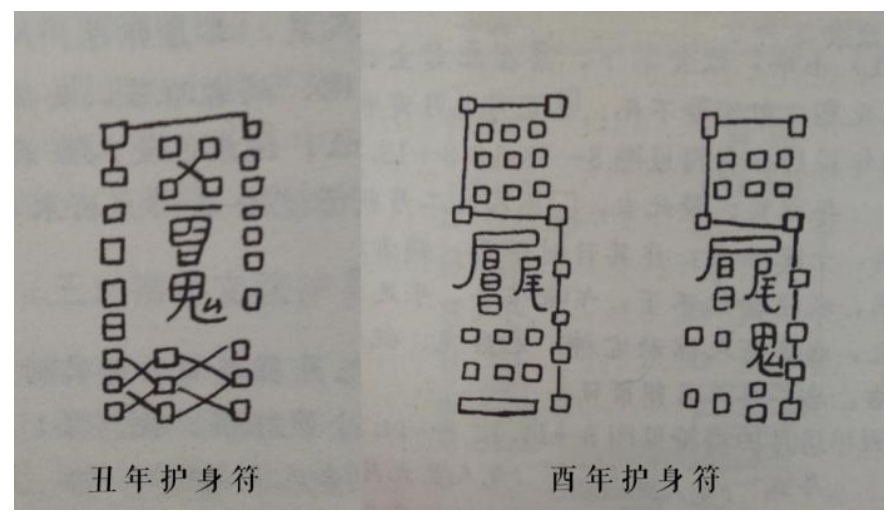

Fig. 11. The animal year amulet.

\section{Mystery Sense of Rune}

Mystery sense of rune is the result of evolution of "symbol" and "incantation". The paper focuses on the mystery sense of "symbol". Characters on the rune are abstract. On the basis of the original characters, bold transformation, increase and decrease in strokes are adopted, boasting a sense of form and a certain meaning. Common people can't understand the rune except Taoist priests, therefore it has a mystery sense. Rune is usually related to pharmacology in medical science, producing the department of incantation and psychology specially set up in the Imperial Hospital. Some runes are

2 Lingbao Immeasurable Person-estimating Top Grade Classics[M]. Volume 37. combined with chemistry, like praying for rain rune using white alum to learn the change in air humidity and predict the weather. Therefore, in some fields, rune has scientific basis and effective results, usually used to pray for good luck and heal illness. However, common people are confused about the rune's profound theory, thus having a mystery sense.

Contemporary Interpreting And Innovative Design Of Rune Under Cultural Creative Thinking

As the representative of Taoism calligraphy, rune has special aesthetic value and a mystery sense. Research on character modeling features of rune provides the basis for its contemporary interpreting and development of cultural products. Just like characters' framework, its framework provides guidance for re-design of rune characters in direction. Its stroke features research equals to filling the skeleton with blood and flesh as details and its combination of pictures and characters makes the newly designed characters full and beautiful. Analysis on its symbolic features endows it with a new soul. Enrichment of symbolic meaning avoids loss of traditional cultural sense in subsequent cultural products. Nowadays, cultural products design falls into an awkward situation: becoming a functional and practical product which is ugly and abandoned by the market or becoming a product with concise appearance and without traditional culture sense due to the blindly pursuit of western formalism. Therefore, the following things are of great importance: how to keep the peculiarities of rune characters, introduce contemporary cultural creative thinking and combine with proper contemporary interpreting and innovative design.

\section{Design Principle of Sticking to the Leading Role of \\ Practicability and Searching for New Carriers}

Integration of traditional culture into product design is never copy and mechanical apply of the original pattern, or, in other words, resulting in an "ostentation" vacant shell. Instead, extraction and new interpreting should be done according to features of product and the meaning carried by traditional culture. Rune culture is rich in form sense and interesting in meaning. Cultural product development of rune should use modern techniques to extract and purify and design interesting character contents with features of the times and search for products fitting the meaning on the basis of research on rune's character modeling features. In ancient times, people usually fold up the written paper rune to carry with or written the rune on peach wood to adorn the body for the purpose of praying for good luck and exorcising evil spirits. It becomes a new way of cultural product development to consider how to get inspiration from ancient people using the rune to pray for good luck and drive out evil spirits and satisfy current people's requirement of product function. Take "Fig. 12", re-design of ornaments and pendants, as an example. Associate the way to pray for good luck and drive out evil spirits through writing the rune on the peach wood and take ornaments as the carrier to conduct development of relevant cultural products. Ancient people usually use sandalwood or peach wood as objects to drive out evil spirits. In terms of material, compared with peach wood, sandalwood is more solid with longer fragrance and richer color and luster. Therefore sandalwood inlaid with gold or silver makes ornaments more fashionable and beautify 
at the same time of achieving the efficacy of driving out evil spirits. Based on retaining lettering features of rune, re-design of characters is done in combination with modern popular cyber words, endowing the presenting mode of characters with contemporaneity. Some runes aim to heal disease and drive out disasters. As to the efficacy of beautifying the skin, based on pharmacology principles, prescription ingredients are ground into powder which is added into the pigment for writing. Then the written rune is burnt into ashes and drunk by the patient, who gets better through change in psychological state, mostly due to psychological comfort and hint. In "Fig.13", the facial mask looks for common points in rune's efficacy to beautify the skin and modern facial mask design, boasting more practicability. The fashionable words of "white, rich and beautiful" added to facial mask design reflect facial mask's skin whitening efficacy and the effect female desires to achieve. Through combination with rune's lettering features, the re-designed characters are interesting with a sense of form. The facial mask satisfies practicability in a ridiculing and interesting way and caters to requirements of young female searching for spoof and loving fresh things.
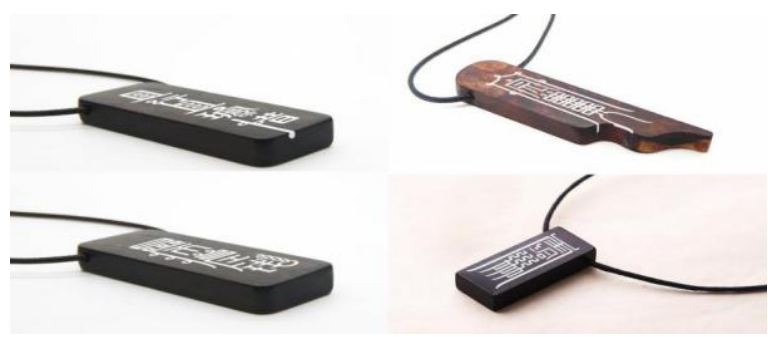

Fig. 12. Re-design effect drawing 1 of ornaments.

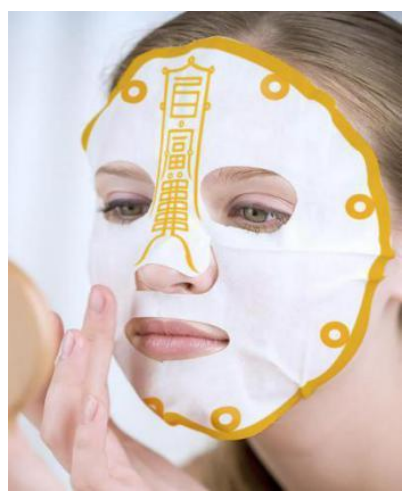

Fig. 13. Re-design effect drawing of facial mask.

\section{E. Design Principle of Sticking to the Auxiliary Role of Interestingness and Combining with Emerging Material}

At present, the development of rune character is very limited. One important reason is people's awe and taboo mentality towards runes for a long time, which can be solved through interesting design. As an art of necromancy, rune forms a mystery sense, one reason of which is that rune smartly uses physical and chemical reaction with certain scientific bases. One good example is folk people using a praying for rain rune to predicate the weather. White alum is used as the pigment to draw the rune. Characters on the rune would appear or disappear according to changes in air humidity, thus predicating the weather. Combine the ancient people's way to predicate the weather through chemical principle with modern umbrella design and use modern emerging materials to make cultural products interesting. In design of umbrella, ancient people's wisdom of using chemical reaction to predicate the weather is borrowed and emerging technological material -- water evident slurry is used to replace white alum. Water evident slurry is a kind of macromolecule printing materials. It is colorless and transparent when dry and boasts patterns when air humidity gets high. It is greatly waterproof with a water fastness of four grade. Therefore it is very suitable for printing of umbrella cloth cover coming into contact with water frequently. Through printing the redesigned rune characters on umbrella cloth with water evident slurry, predicating the weather becomes interesting. As shown in "Fig.14", it is a simple and unadorned umbrella without any pattern on sunny days and characters of "raining" would appear on rainy days, making the umbrella interesting and acting in cooperation with features of ancient praying for rain rune.

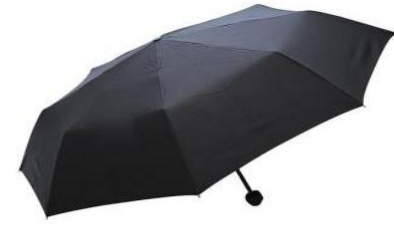

Sunny

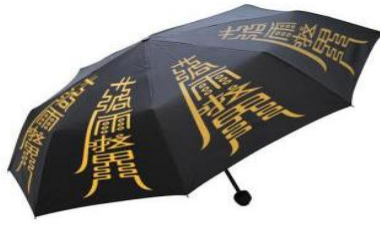

Cloudy
Fig. 14. Re-design effect drawing of umbrella.

\section{F. Design Principle of Sticking to the Complementing Role of Beauty and Applying New Forms}

The good and bad cultural products are intermingled on the current market and many products are practical and interesting, yet lack beauty and fail to win over consumers at first time, thus making it difficult for them to show their practical and interesting features. Therefore, interesting, beautiful and practical are of prime importance to development of cultural products. Rune characters' framework, aesthetic features and symbolic nature endow them with unique beauty and lasting appeal. As shown in "Fig. 15", on the basis of concluding lettering features of characters, evident features of rune characters are extracted to develop characters and conduct standard design of rune characters in various styles and varieties, the overall style solemn and just without losing features. Therefore, development of cultural products of rune characters also needs to satisfy current aesthetic trends, obey the design principle of interesting, beautiful and practical and find a broader consumption market, thus contributing to inheritance and transformation of traditional folk custom culture. 


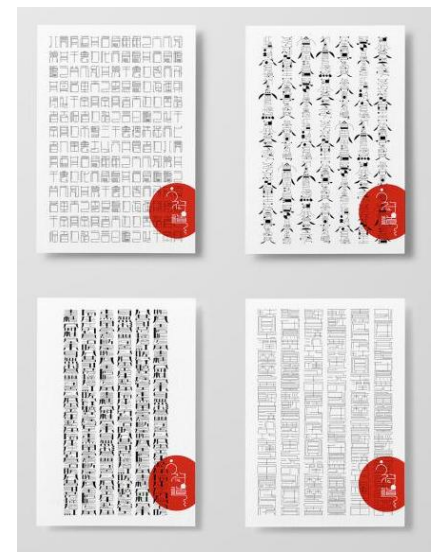

Fig. 15. Re-design of rune character.

\section{CONCLUSION}

Rune characters boast unique modeling with distinctive manifestation form, various carriers and rich meanings, being one of the representatives of traditional Chinese cultural symbol. Through research and exploration on rune characters, it is not difficult to find out that to conduct innovative design of traditional folk custom culture like rune characters, first analysis and conclusion on modeling features of characters or patterns should be done, "obtaining its form" on the basis of understanding; second, in-depth exploration on the cultural background and application scenarios and searching for the common point with modern products need to be done in order to inherit culture and realize "extension of the meaning". At the same time, emerging materials should be used to achieve integration innovation, exploring more possibilities of cultural product design through the way of "conveying the meaning".

\section{REFERENCES}

[1] Li Zehou. Aesthetics Collection[M]. Shanghai: Shanghai Literature and Art Publishing House, 1980, Page 532.

[2] Ulrich Eppinger (American). Product Design and Development[M], Translated by Yang Qing etc. Beijing: China Machine Press, 2015, Page 2

[3] Liu Xiaoming. Chinese Rune Culture Spectacle[M]. Nanchang: Hundred Flower Pond Literature and Art Publishing House, 2010, Page 8-14.

[4] Yao Zhouhui. Mysterious Rune Incantation[M]. Nanning: Guangxi People's Publishing House, 2007, Page 1-2.

[5] Wu Fenggao. Rune Character Formal Change Research[D]. Sichuan: Jiangxi Normal University, 2010, Page 24-29.

[6] Li Juntao. Yun Zhuan Tian Shu: Taoism Rune Art's Enlightenment on Modern Visual Design[D]. Sichuan: Sichuan University, 2006, Page 7. 